D FUNGAL PATHOGENESIS

\section{Glued for protection}

DOI:

10.1038/nrmicro1649

URLs

Aspergillus fumi-

gatus

http://www.ncbi.nlm. nih.gov/entrez/query. $\mathrm{fcgi}$ ? $\mathrm{db}=$ genomeprj $\& \mathrm{cmd}=$ Retrieve\&do $\mathrm{pt}=$ Overview\&list_ uids $=9521$

A study just published in Cellular Microbiology has probed the three-dimensional structure of the mycelia of the deadly fungal pathogen Aspergillus fumigatus for the first time. The authors reveal that hyphae are stuck together with a hydrophobic glue to form the fungal balls (aspergilloma) that characterize the pulmonary diseases caused by this pathogen. The glue might be important in protecting the fungus from immune defences and drug action.

A. fumigatus causes often-lethal opportunistic invasive lung infections in immunocompromised individuals and allergies in asthma and cystic fibrosis sufferers. In the laboratory, aspergilli are usually grown in shake flasks or fermentors - a convenient way to harvest biomass for experiments. But in the lungs inhaled spores (conidia) germinate, producing aerial hyphae that clump to form fungal balls.

Beauvais et al. realized that the growth of fungal balls is most closely mimicked in the laboratory by growing aspergilli on solid agar media. By comparing features of mycelia grown on solid media and in liquid culture they hoped to gain insights into the pathogenesis of A. fumigatus. Perhaps surprisingly, biomass accumulated more quickly and to higher dry weights when A. fumigatus was grown on solid media. All the cultures were equally sensitive to imidazoles and caspofungins, but polyene drugs, especially nystatin, killed far fewer cells in cultures that were grown on solid media compared with liquidgrown cells.

Accordingly, Beauvais et al. investigated the morphology of mycelia grown in different condi- tions to assess why drugs might have different effects depending on culturing conditions. Using scanning and transmission electron microscopy they noted that aerial hyphae grown on solid media were covered in a granular extracellular slime that was absent from hyphae grown in flasks. In a mouse model of aspergillosis, hyphae in the lungs were coated with slime too, indicating a potential role for the slime in pathogenesis. Mycelial mats grown on solid media were also considerably more hydrophobic than those grown in shake flasks, probably because hyphae are encased in slime. Biochemical tests defined the slime matrix composition: galactomannan, $\alpha 1,3$-glucans, monosaccharides, polyols, melanin and proteins, including antigens and hydrophobins.
The aerial mycelial mat formed by A. fumigatus has intercellular channels and an extracellular matrix, two hallmarks of biofilms. As nystatin was not as effective on the slime-covered fungal balls, the next step for this research is determining whether the glue that binds the balls together in the lungs is the same as the glue that coats laboratory-grown cells.

Microbiologists are striving to understand how organisms grow and interact in communities in natural environments. This study shows that going back to basics and finding the laboratory model that best mimics the growth of a pathogen in the host can significantly affect our understanding of pathogenesis. Following this approach should help researchers to further dissect pathogenic strategies and design more effective therapeutic interventions.

Susan Jones

ORIGINAL RESEARCH PAPER Beauvais, A. et al. An extracellular matrix glues together the aerial-grown hyphae of Aspergillus fumigatus. Cell Microbiol. 16 Feb 2007 (doi:10.1111/j.14625822.2007.00895.x)

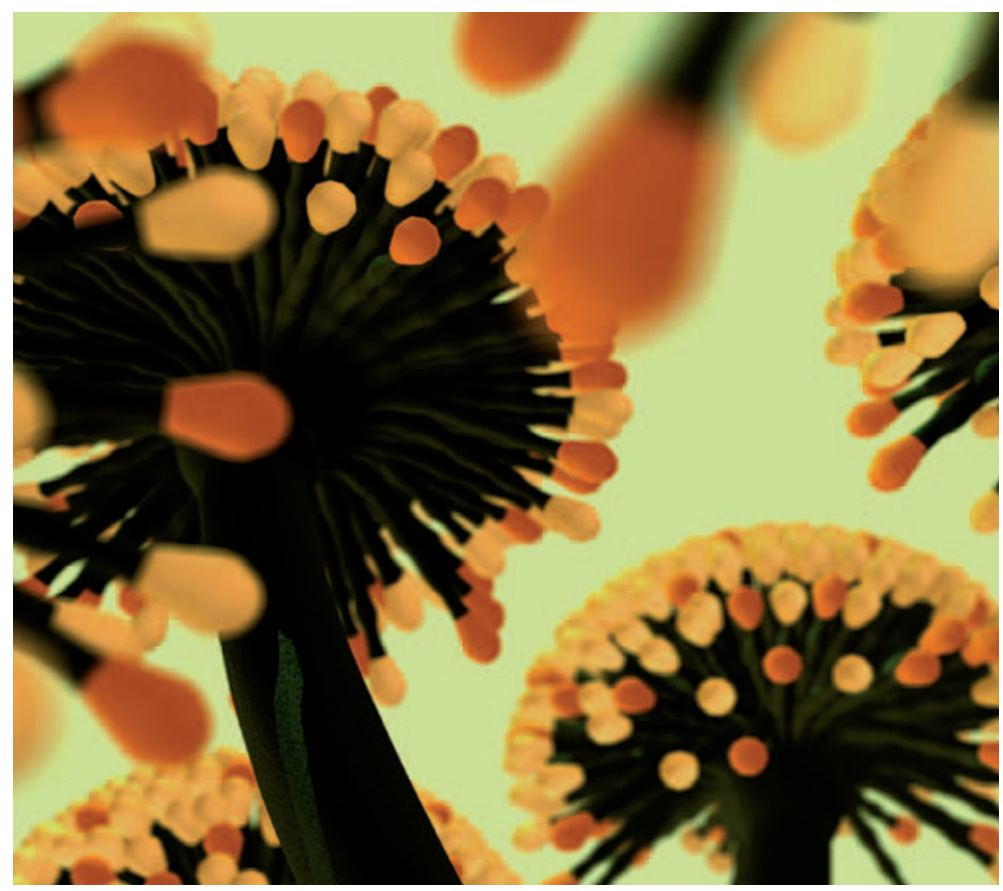

